\title{
Error Analysis of Phase-Integral Methods. I. General Theory for Simple Turning Points
}

\author{
F. W. J. Olver
}

(July 1, 1965)

An approximate general solution of the differential equation

$$
d^{2} w / d z^{2}=f(z) w
$$

is

$$
w \approx A f^{-1 / 4} \exp \left(\int f^{1 / 2} d z\right)+B f^{-1 / 4} \exp \left(-\int f^{1 / 2} d z\right)
$$

To represent a particular solution throughout a given complex domain, differing pairs of values of the arbitrary constants $A$ and $B$ are generally needed in various subdomains. Strict error bounds are established in this paper for the coefficients in the linear equations connecting the pairs of values of $A$ and $B$, in the case in which the $z$-domain under consideration is unbounded and contains a simple zero and no singularities of $f(z)$.

The results are used to place the "WKBJ" method on a firm mathematical foundation.

Key Words: Differential equations, asymptotics, phase-integral, WKBJ method, turning point, connection formulas, error bounds.

\section{Introduction}

A form of solution of the differential equation

$$
\frac{d^{2} w}{d z^{2}}=f(z) w
$$

which is frequently used in applied mathematical problems, is given by

$$
w \approx A f^{-1 / 4} \exp \left(\int f^{1 / 2} d z\right)+B f^{-1 / 4} \exp \left(-\int f^{1 / 2} d z\right)
$$

in which $A, B$ are arbitrary constants. This is commonly referred to as the Liouville-Green approximation, the $W K B$ or $W K B J$ approximation, or the phase-integral solution. A necessary condition for the applicability of the approximation is that $f^{-1 / 4}$ be a bounded, slowly varying function of $z[1]{ }^{1}$ This condition automatically excludes from the region of validity most kinds of singularity of $f$, and also zeros of $f$, the so-called turning or transition points of the differential equation.

A further condition concerns the monotonicity of the function Re $\int f^{1 / 2} d z$ (see [1], sec. 8, and sec. 4 , below). This requirement has the effect of dividing the $z$-region under consideration, $\mathbf{D}$, say, into a number of subregions, in each of which a different combination of values of $A$ and $B$ in (1.02) has to be used in order to represent the same solution of (1.01). This is, of course, an example of the Stokes phenomenon. The linear equations linking the values of $A$ and $B$ in one subregion with those in another are called the connection formulas or Stokes equations. The purpose of this paper is to determine the connection formulas in the case in which $\mathbf{D}$ contains no singularity of $f(z)$, and just one simple turning point, $z_{0}$, say.

${ }^{1}$ Figures in brackets indicate the literature references at the end of this paper. 
Two principal methods are available for attacking this problem. The first, applicable when $f(z)$ is real on the real axis, starts with the Liouville-Green approximation in real exponential form on one side of $z_{0}$, then traces its behavior along a semicircle in the complex plane to the real axis on the other side of $z_{0}$. The condition that the solution must be real when $z$ is real suffices to determine the main features of the appropriate connection formula. This method was originated by Zwaan [2], and has been developed further by Kemble [3] and Furry [4].

The second method consists of using approximations to the solutions in terms of Airy functions (or equivalently Bessel functions of order one-third), the region of validity of which includes $z_{0}$. Away from $z_{0}$ the Airy functions are approximated by their asymptotic forms for large argument, leading immediately to the Liouville-Green forms. In the original version [5]-[7], the Airy-function representations were constructed by replacing $f(z)$ by a linear approximation in the neighborhood of $z_{0}$; this is, perhaps, the true "WKBJ method." In more recent work [8]-[13] more powerful uniform approximations in terms of Airy functions have been used to derive the connection formulas.

Hitherto, the method of Zwaan has not been put on a sound mathematical footing: writing in 1934 Langer [14] commented ". . . an estimation of the relative error involved in the result is almost impossible..." The treatment of the Airy-function procedure has been more satisfactory. By supposing that $f(z)$ contains as multiplicative factor (the square of) a large parameter $u$, say, it is shown in the references cited that the connection formulas are asymptotically correct as $|u| \rightarrow \infty$.

In the present paper we give rigorous analytical derivations of both methods, and supply explicit bounds for the errors in the connection formulas (thus overcoming one of Langer's objections to Zwaan's method). Since we are dealing with bounds there is no need for the explicit introduction of a parameter $u$ in the theory: asymptotic properties in applications can be regarded as immediate consequences of the form of error bound. ${ }^{2}$ We also dispense with the requirement, in Zwaan's method, that $f(z)$ be real on the axis.

The starting points of our investigation are the theories of error bounds for the Liouville-Green and uniform Airy approximations which have been developed by the writer in [1] and [15].

Since the present paper and its sequel [19] were written, there has appeared a monograph on the same subject by N. Fröman and P. O. Fröman [20]. Although the objective of these authors is essentially the same as that of the present writer, the methods used and results achieved are sufficiently different to warrant publication of this paper and [19] in substantially their original forms. Some comparisons with the results of [20] are given in section 11.

\section{First Error Theorem}

We begin with the differential equation

$$
\frac{d^{2} w}{d z^{2}}=\{1+f(z)\} w
$$

in which $f(z)$ is a regular (holomorphic) function in a simply-connected complex $z$-domain $\mathbf{D}$.

Theorem 1. Equation (2.01) has solutions $w_{j}(z)(j=1,2)$ regular in $\mathbf{D}$, and depending on arbitrary fixed points $a_{j}$ of $\mathbf{D}$, such that

$$
w_{j}(z)=e^{ \pm z}\left\{1+\epsilon_{j}(z)\right\}
$$

where

$$
\left|\epsilon_{j}(z)\right|,\left|\epsilon_{j}^{\prime}(z)\right|,\left|\epsilon_{j}(z) \pm \epsilon_{j}^{\prime}(z)\right| \leqslant \exp \left\{\mathscr{V}_{\mathscr{P}_{j}}(G)\right\}-1
$$

when $z \in \mathbf{H}_{j}$.

${ }^{2}$ In the course of a valuable historical introduction io phase integral methods, Heading ([13], p. 8) critizes other writers, particularly Budden [12], for failing to standardize a large parameter in their analysis. The theory of error bounds presented in this paper provides a broader basis for justifying the work of these writers. 
In this result, the upper signs are to be taken when $j=1$, and the lower signs when $j=2$. The regions of validity $\mathbf{H}_{j}(j=1,2)$ comprise the aggregate of those points $z$ for which there exists a path $\mathscr{P}_{j}$ joining $z$ with $a_{j}$ and lying entirely in $\mathbf{D}$, with the properties:

(i) $\mathscr{P}{ }_{j}$ consists of a finite number of Jordan ares, each having a parametric equation of the form $t=t(\tau)$ with $t^{\prime \prime}(\tau)$ continuous and $t^{\prime}(\tau)$ nonvanishing.

(ii) Re $t$ is monotonic nonincreasing $(j=1)$ or monotonic nondecreasing $(j=2)$, as $t$ traverses $\mathscr{P}_{j}$ from $z$ to $a_{j}$.

Lastly, $\mathscr{V}_{\mathscr{P}_{j}}(G)$ denotes the variation along $\mathscr{P}_{j}$ of the function

$$
G(z)=\int f(z) d z
$$

thus

$$
\mathscr{W}_{i \mathscr{p}_{j}}(G)=\int_{z}^{a_{j}}|f(t) d t|
$$

Theorem 1 is essentially a special case of Theorem 7 of [1]. We record the main steps of the proof, however, since they are needed in the derivation of further properties of the error terms in the next section.

For illustration, we take $j=1$. From (2.01) and (2.02) we find that

$$
\epsilon_{1}^{\prime \prime}(z)+2 \epsilon_{1}^{\prime}(z)=f(z)\left\{1+\epsilon_{1}(z)\right\} .
$$

Therefore $\epsilon_{1}(z)$ satisfies the integral equation

$$
\epsilon_{1}(z)=\frac{1}{2} \int_{u_{1}}^{z}\left\{1-e^{2(t-z)}\right\}\left\{1+\epsilon_{1}(t)\right\} f(t) d t,
$$

in which the path of integration is taken to be $\mathscr{P}_{1}$. The solution of this equation is constructed as a convergent series

$$
\epsilon_{1}(z)=\sum_{n=1}^{\infty} l_{1, n}(z)
$$

in which $l_{1,0}(z)=1$, and

$$
l_{1, n}(z)=\frac{1}{2} \int_{u_{1}}^{z}\left\{1-e^{2(t-z)}\right\} f(t) l_{1, n-1}(t) d t \quad(n \geqslant 1)
$$

In consequence of Condition (ii) on $\mathscr{P}_{1}$ we have

$$
\left|e^{2(t-z)}\right| \leqslant 1
$$

and by substituting this bound in (2.09) we readily establish by induction that

$$
\left|l_{1, n}(z)\right| \leqslant\left\{\mathscr{V}_{a_{1}, z}(G)\right\}^{n} / n ! \quad(n \geqslant 0),
$$

showing that the series (2.08) is uniformly convergent. In order that solutions of the integral equation (2.07) also represent solutions of the differential equation (2.06), some restrictions are needed on the path $\mathscr{P}_{1}$. Those given in (i) above suffice (compare [16], pp. 491-492).

The first of the inequalities (2.03) now follows by summation of (2.11). The other two are derived similarly from the differentiated form of (2.09). 


\section{Further Properties of the Error Terms}

For conciseness, we shall refer to a path $\mathscr{P}_{j}$ which fulfills Condition (i) of section 2 and is such that $\operatorname{Re} t$ is monotonic as $t$ traverses $\mathscr{P}_{j}$ as a progressive path. If the monotonicity is strict everywhere, we say that the path is strictly progressive. It may be noted, incidentally, that Condition (i) is quite weak, and can be fulfilled, for example, by any finite chain of straight lines and circular arcs.

The condition that $\mathbf{D}$ be simply connected, mentioned in the opening paragraph of section 2 , merely ensures that each $w_{j}(z)$ is single-valued in D. For a multiply connected domain, each branch of $w_{j}(z)$ satisfies (2.02) and (2.03) whenever a progressive path can be found in $\mathbf{D}$ joining $z$ with $a_{j}$.

In section 2 we tacitly assumed that the points $a_{j}$ are finite. Theorem 1 still holds, however, if $\mathbf{D}$ is unbounded and the $a_{j}$ are points at infinity on progressive paths $\mathscr{L}_{j}$, provided that $\mathscr{P}_{j}$ coincides with $\mathscr{L}_{j}$ as $a_{j}$ is approached and that the variations (2.05) converge. The convergence of the infinite integrals (2.09) is then an immediate consequence of the inequality (2.11).

A situation of importance in the later sections occurs when $a_{1}$ and $a_{2}$ are distinct points at infinity, and can be joined by a progressive path $\mathscr{P}$ lying in $\mathbf{D}$. From (2.03) it is seen that $\epsilon_{1}(z)$ and $\epsilon_{1}^{\prime}(z)$ both vanish as $z \rightarrow a_{1}$ along $\mathscr{P}$; indeed we may regard these properties as the boundary conditions defining $w_{1}(z)$. What happens, however, to $\epsilon_{1}(z)$ and $\epsilon_{1}^{\prime}(z)$ as $z \rightarrow a_{2}$ ?

THEOREM 2. If $|\operatorname{Re} z| \rightarrow \infty$ as $z \rightarrow a_{1}$ and as $z \rightarrow a_{2}$, then

$$
\begin{gathered}
\epsilon_{1}(z) \rightarrow \text { a constant } \epsilon_{1}\left(a_{2}\right), \text { say, and } \epsilon_{1}^{\prime}(z) \rightarrow 0 \text { as } z \rightarrow a_{2}, \\
\epsilon_{2}(z) \rightarrow \text { a constant } \epsilon_{2}\left(a_{1}\right), \text { say, and } \epsilon_{2}^{\prime}(z) \rightarrow 0 \text { as } z \rightarrow a_{1}, \\
\epsilon_{1}\left(a_{2}\right)=\epsilon_{2}\left(a_{1}\right)
\end{gathered}
$$

and

$$
\left|\epsilon_{1}\left(a_{2}\right)\right| \leqslant \frac{1}{2}\left[\exp \left\{\mathscr{f}_{\mathscr{Q}}(G)\right\}-1\right]
$$

To prove these results, let

$$
\alpha_{1, n}(z)=\int_{l_{1}}^{z} f(t) l_{1, n-1}(t) d t, \quad \beta_{1, n}(z)=\int_{l_{1}}^{z} e^{2(t-z)} f(t) l_{1, n-1}(t) d t .
$$

Then from (2.08) and (2.09), we have

$$
\epsilon_{1}(z)=\frac{1}{2} \sum \alpha_{1, n}(z)-\frac{1}{2} \sum \beta_{1, n}(z), \quad \epsilon_{1}^{\prime}(z)=\Sigma \beta_{1, n}(z)
$$

the summations extending in each case from $n=1$ to $n=\infty$. Since by hypothesis $\int_{a_{1}}^{a_{2}}|f(t) d t|$ converges, it follows that for any given positive number $\delta$ which is less than the value of this doubly infinite integral there exists a point $z^{\prime}$ on $\mathscr{P}$ such that

$$
\int_{z^{\prime}}^{a_{2}}|f(t) d t|=\delta
$$

Let $z$ be any point of $\mathscr{P}$ between $z^{\prime}$ and $a_{2}$ (fig. 1). Then by splitting the integration range in the second of (3.04) at $z^{\prime}$, and applying (2.10), (2.11), we find that

$$
\left|\beta_{1, \mu}(z)\right| \leqslant\left|e^{2\left(z^{\prime}-z\right)}\right| \frac{\left\{\|_{a_{1}, z^{\prime}}(G)\right\}^{n}}{n !}+\frac{\left\{\psi_{a_{1}, a_{2}}(G)\right\}^{n-1}}{(n-1) !} \delta \text {. }
$$


Therefore

$$
\left|\Sigma \beta_{1, n}(z)\right| \leqslant\left|e^{2\left(z^{\prime}-z\right)}\right|\left[\exp \left\{\mathscr{\gamma}_{a_{1}, z^{\prime}}(G)\right\}-1\right]+\delta \exp \left\{\gamma_{a_{1}, a_{2}}(G)\right\}
$$

With the assumed conditions, $e^{2\left(z^{\prime}-z\right)}$ vanishes as $z \rightarrow a_{2}$. Hence $\Sigma \beta_{1, n}(z)$ also vanishes, that is, $\epsilon_{1}^{\prime}(z) \rightarrow 0$.

Next, we have

$$
\left|\alpha_{1, n}(z)-\alpha_{1, n}\left(z^{\prime}\right)\right|=\left|\int_{z^{\prime}}^{z} f(t) l_{1, n-1}(t) d t\right| \leqslant \frac{\left\{y \omega_{1, z}(G)\right\}^{n}-\left\{\omega_{1, z^{\prime}}(G)\right\}^{n}}{n !},
$$

and so

$$
\left|\Sigma \alpha_{1, n}(z)-\Sigma \alpha_{1, n}\left(z^{\prime}\right)\right| \leqslant \exp \left\{\gamma_{\omega_{1}, z}(G)\right\}-\exp \left\{\psi_{\mu_{1}, z^{\prime}}(G)\right\}
$$

The right side of this inequality vanishes as $z$ and $z^{\prime}$ tend independently to $a_{2}$, therefore $\sum \alpha_{1, n}(z)$ tends to a constant limiting value. The proof of the first line of (3.01) is now complete. The second line follows by symmetry.

To prove (3.02), we construct the Wronskian from (2.02):

$$
\mathscr{W}_{\left(w_{2}, w_{1}\right)}=\left\{1+\epsilon_{2}(z)\right\}\left\{1+\epsilon_{1}(z)+\epsilon_{1}^{\prime}(z)\right\}+\left\{1+\epsilon_{1}(z)\right\}\left\{1+\epsilon_{2}(z)-\epsilon_{2}^{\prime}(z)\right\} .
$$

From (3.01) we see that this reduces to $2\left\{1+\epsilon_{2}\left(a_{1}\right)\right\}$ at $z=a_{1}$, and $2\left\{1+\epsilon_{1}\left(a_{2}\right)\right\}$ at $z=a_{2}$. Since the Wronskian is constant, (3.02) follows.

Lastly, we derive from (2.11) and (3.04)

$$
\left|\alpha_{1, n}\left(a_{2}\right)\right| \leqslant\left\{\mathscr{V}_{a_{1}, a_{2}}(G)\right\}^{n} / n !
$$

The improved form (3.03) of the main result (2.03) for the special value $z=a_{2}$ now follows immediately from (3.05).

\section{Basic Error Theorem for the Liouville-Green Approximation}

We now proceed to apply the results of the preceding two sections to the differential equation (1.01). A useful generalization is achieved on replacing the coefficient of $w$ in this equation by the sum of two functions $f(z)$ and $h(z)$, say. In applications the second function $h(z)$ will either be zero, or small, in some sense, compared with $f(z)$ (cf. [1], sec. 4(ii)). From the standpoint of the error analysis, however, the relative magnitudes of $f(z)$ and $h(z)$ are immaterial.

Thus we consider the differential equation

$$
\frac{d^{2} w}{d z^{2}}=\{f(z)+h(z)\} w
$$


in which $f(z)$ and $h(z)$ are regular in an unbounded domain $\mathbf{D}$, not necessarily simply connected, and $f(z)$ is nonvanishing. The Liouville transformation

$$
\xi=\xi(z)=\int\{f(z)\}^{1 / 2} d z, \quad W=\{f(z)\}^{1 / 4} w,
$$

throws (4.01) into the form

$$
d^{2} W / d \xi^{2}=\{1+\varphi(\xi)\} W
$$

in which

$$
\varphi(\xi)=\frac{h(z)}{f(z)}+\frac{4 f(z) f^{\prime \prime}(z)-5\left\{f^{\prime}(z)\right\}^{2}}{16\{f(z)\}^{3}}=\frac{h}{f}-\frac{1}{f^{3 / 4}} \frac{d^{2}}{d z^{2}}\left(\frac{1}{f^{1 / 4}}\right) .
$$

Equation (4.03) is of the form (2.01). The $z$-domain $\mathbf{D}$ is mapped on a $\xi$-domain $\Delta$, say, and $\varphi(\xi)$ is regular in $\Delta$. By applying Theorem 1 and then restoring the original variables we establish the following:

THEOREM 3. Let $z=c$ be an arbitrary point at infinity in $\mathbf{D}$ such that $|\operatorname{Re} \xi(c)|=\infty$, and let the branch of $\xi(z)$ be chosen in such a way that $\operatorname{Re} \xi(z)$ is positive as $c$ is approached. Then the differential equation (4.01) has a regular solution, given by

$$
w(z)=\{f(z)\}^{-1 / 4} e^{-\xi(z)}\{1+\epsilon(z)\}
$$

where

$$
|\epsilon(z)|,\left|f^{-1 / 2} \epsilon^{\prime}(z)\right|,\left|\epsilon(z)-\left\{\epsilon^{\prime}(z) / \xi^{\prime}(z)\right\}\right| \leqslant \exp \left\{\mathscr{V}_{z, c}(F)\right\}-1 \quad(z \in \mathbf{H}(c)),
$$

and

$$
F(z)=\int \varphi(\xi) d \xi=\int\left\{\frac{h}{f^{1 / 2}}-\frac{1}{f^{1 / 4}} \frac{d^{2}}{d z^{2}}\left(\frac{1}{f^{1 / 4}}\right)\right\} d z
$$

The region of validity $\mathbf{H}(c)$ comprises those points $z$ which can be joined in $\mathbf{D}$ to $c$ by a " $\xi$-progressive" path $\mathscr{P}$, that is, one whose map in the $\xi$-plane is progressive (sec. 3 ). All branches in (4.05) to (4.07) are continuous along $\mathscr{P}$, that of $f^{1 / 2}$ being the square of $f^{1 / 4}$. The variation in (4.06) is taken over $\mathscr{P}$; naturally we assume that it converges at $c$, otherwise (4.06) is meaningless.

\section{Fundamental Connection Formula for a Simple Turning Point}

In this section we suppose that $f(z)$ and $h(z)$ are regular in an unbounded, simply connected domain $\mathbf{D}$, and that $f(z)$ has just one zero in $\mathbf{D}$, a simple zero at an interior point $z_{0}$, say. We define $\xi(z)$ by (4.02), fixing the arbitrary lower integration limit at $z_{0}$ :

$$
\xi(z)=\int_{z_{0}}^{z}\{f(t)\}^{1 / 2} d t
$$

Clearly $\xi(z)$ is an analytic function in $\mathbf{D}$ whose only singularity is a branch point at $z_{0}$. If the Taylor expansion of $f(z)$ in the neighborhood of $z_{0}$ is denoted by

$$
f(z)=\sum_{n=1}^{\infty} f_{n}\left(z-z_{0}\right)^{n}
$$


then

$$
\xi(z)=\frac{2}{3} f_{1}^{1 / 2}\left(z-z_{0}\right)^{3 / 2}\left\{1+O\left(z-z_{0}\right)\right\} .
$$

The set of points in the $z$-plane for which

$$
\operatorname{Re} \xi(z)=0
$$

we call the principal curves ${ }^{3}$ associated with the turning point $z_{0}$. Using the theory of conformal mapping, we see that each principal curve is a regular Jordan arc which can terminate only at $z_{0}$ or at the boundary of $\mathbf{D}$. Moreover, no principal curve can intersect itself or any other principal curve except at $z_{0}$. Equation (5.03) shows that three principal curves emerge from $z_{0}$, inclined there at angle $2 \pi / 3$ with each other; we denote them by $\mathscr{C}_{1}, \mathscr{C}_{2}, \mathscr{C}_{3}$, enumerated in the positive rotational sense. These and other principal curves divide $\mathbf{D}$ into a number of subdomains. Those with $z_{0}$ on their boundary we call the principal subdomains, and denote them by $\mathbf{D}_{1}, \mathbf{D}_{2}, \mathbf{D}_{3}$, as indicated on figure 2. Clearly each $\mathbf{D}_{j}$ is itself simply connected. For terminological convenience in what follows we shall enumerate $j$ with modulo 3 ; thus $\mathbf{D}_{0} \equiv \mathbf{D}_{3}, \mathbf{D}_{4} \equiv \mathbf{D}_{1}$.

We define $\xi_{j}(z)(j=1,2,3)$ to be that branch of $\xi(z)$ which is continuous in $\mathbf{D}$ cut along the corresponding $\mathscr{C}_{j}$, and whose real part is positive in $\mathbf{D}_{j}$ and negative in the other two principal subdomains. Analytic continuation across the cut $\mathscr{C}_{j}$ is expressed by

$$
\xi_{j}\left\{\left(z-z_{0}\right) e^{ \pm 2 \pi i}\right\}=-\xi_{j}\left(z-z_{0}\right)
$$

compare (5.03). We define $g(z)$ to be the four-valued function

$$
g(z)=\{f(z)\}^{-1 / 4} .
$$

Its analytic continuation is expressed by

$$
g\left\{\left(z-z_{0}\right) e^{ \pm 2 \pi i}\right\}=\mp i g\left(z-z_{0}\right) .
$$

We denote by $g_{j}(z)(j=1,2,3)$ an arbitrary constant multiple of any branch of $g(z)$ which is continuous in $\mathbf{D}$ cut along the corresponding $\mathscr{C}_{j}$. In order to achieve a symmetric form of final result, we stipulate that these multiples must be chosen in such a way that

$$
g_{j}(z)=e^{\pi i / 6} g_{j-1}(z) \quad\left(z \in \mathbf{D}_{j-1} \cup \mathbf{D}_{j}\right) .
$$

${ }^{3}$ Also called anti-Stokes lines [12], [13].

Figure 2. Principal subdomains $\mathbf{D}_{j}$.

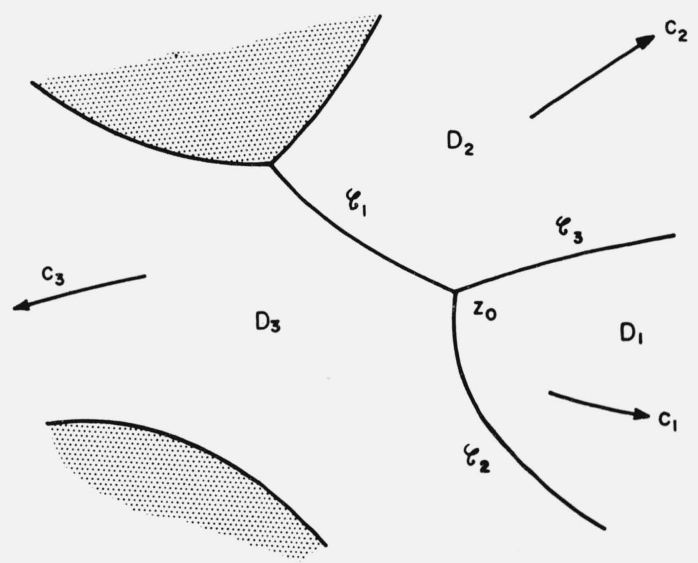


The self-consistency of this equation for $j=1,2,3$ follows from (5.07).

Let us suppose that each $\mathbf{D}_{j}$ is unbounded, and that $c_{j}$ is an arbitrary point at infinity in $\mathbf{D}_{j}$ such that $\left|\operatorname{Re} \xi\left(c_{j}\right)\right|=\infty$. Excluding the point $z_{0}$ from $\mathbf{D}$, and then applying Theorem 3 of section 4 , we find that eq (4.01) has solutions $w_{j}(z)(j=1,2,3)$, given by

$$
w_{j}(z)=g_{j}(z) e^{-\xi_{j}(z)}\left\{1+\epsilon_{j}(z)\right\},
$$

where

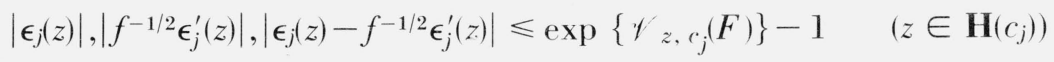

and $F$ is defined by (4.07). In (5.10) the branch of $f^{-1 / 2}$ is $1 / \xi_{j}^{\prime}(z)$, and $\mathbf{H}\left(c_{j}\right)$ is the set of $z$-points which can be joined to $c_{j}$ by a $\xi$-progressive path $\mathscr{P}_{j}$ lying in $\mathbf{D}$. (We observe, in passing, that the corresponding principal curve $\mathscr{C}_{j}$ is excluded from $\mathbf{H}\left(c_{j}\right)$, since passage of $\mathscr{P}_{j}$ through $z_{0}$ is not permitted.) $\mathscr{V}_{z, c_{j}}(F)$ denotes the variation over $\mathscr{P}_{j}$ of $F$.

Although each solution $w_{j}(z)$ satisfies (5.09) and (5.10) in only part of $\mathbf{D}$, the theory of linear differential equations shows that each $w_{j}(z)$ exists and is regular throughout the whole of $\mathbf{D}$. Further, there is an identity of the form

$$
A_{1} w_{1}(z)+A_{2} w_{2}(z)+A_{3} w_{3}(z)=0,
$$

in which the $A_{j}$ are independent of $z$. Equation (5.11) is the connection formula for the turning point $z_{0}$, and the values of the $A_{j}$ constitute our primary quest. In order to obtain them, we assume that $\xi$-progressive paths exist in $\mathbf{D}$ linking each pair of reference points $c_{j}, c_{k}$.

Consider (5.11) in $\mathbf{D}_{1}$. Substituting by means of (5.09) and (5.08), and using the relations $\xi_{2}(z)=\xi_{3}(z)=-\xi_{1}(z)$, we derive

$$
A_{1} e^{-\xi_{1}(z)}\left\{1+\epsilon_{1}(z)\right\}+A_{2} e^{(\pi i / 6)+\xi_{1}(z)}\left\{1+\epsilon_{2}(z)\right\}+A_{3} e^{-(\pi i / 6)+\xi_{1}(z)}\left\{1+\epsilon_{3}(z)\right\}=0 .
$$

Now let $z \rightarrow c_{1}$. Then $\epsilon_{1}(z)$ vanishes and, by Theorem $2, \epsilon_{2}(z)$ and $\epsilon_{3}(z)$ tend to constant values $\epsilon_{2}\left(c_{1}\right)$ and $\epsilon_{3}\left(c_{1}\right)$. Also by hypothesis $\operatorname{Re} \xi_{1}(z) \rightarrow+\infty$. Hence we have

$$
e^{\pi i / 6}\left\{1+\epsilon_{2}\left(c_{1}\right)\right\} A_{2}+e^{-\pi i / 6}\left\{1+\epsilon_{3}\left(c_{1}\right)\right\} A_{3}=0 .
$$

Similarly,

$$
\begin{gathered}
e^{-\pi i / 6}\left\{1+\epsilon_{1}\left(c_{2}\right)\right\} A_{1}+e^{\pi i / 6}\left\{1+\epsilon_{3}\left(c_{2}\right)\right\} A_{3}=0, \\
e^{\pi i / 6}\left\{1+\epsilon_{1}\left(c_{3}\right)\right\} A_{1}+e^{-\pi i / 6}\left\{1+\epsilon_{2}\left(c_{3}\right)\right\} A_{2}=0 .
\end{gathered}
$$

The consistency of this set of homogeneous equations for the $A_{j}$ immediately follows from the relation $\epsilon_{j}\left(c_{k}\right)=\epsilon_{k}\left(c_{j}\right)$ obtained from eq (3.02). Solving for the $A_{j}$ and substituting back in (5.11), we arrive at the desired form of the connection formula:

$$
\left\{1+\epsilon_{2}\left(c_{3}\right)\right\} w_{1}(z)+e^{-2 \pi i / 3}\left\{1+\epsilon_{3}\left(c_{1}\right)\right\} w_{2}(z)+e^{2 \pi i / 3}\left\{1+\epsilon_{1}\left(c_{2}\right)\right\} w_{3}(z)=0 .
$$


The error terms are bounded by (compare (3.03))

$$
\left|\epsilon_{j}\left(c_{k}\right)\right| \leqslant \frac{1}{2}\left[\exp \left\{\gamma_{c_{j}, c_{k}}(F)\right\}-1\right]
$$

that is,

in which the variation (or integral) is evaluated along a $\xi$-progressive path linking $c_{j}$ with $c_{k}$.

We summarize this section so far. The assumed conditions on $f(z)$ and $h(z)$ are stated in the opening sentence. The principal subdomains $\mathbf{D}_{j}$ associated with the turning point $z_{0}$ are defined in the second paragraph. Three solutions $w_{j}(z)(j=1,2,3)$ of the differential equation (4.01) are then constructed, given by (5.09) and (5.10). Here $\xi_{j}(z)$ is the branch of the integral (5.01) which has positive real part in $\mathbf{D}_{j} ; g_{j}(z)(j=1,2,3)$ is any set of constant multiples of branches of $\{f(z)\}^{-1 / 4}$ satisfying (5.08); $c_{j}$ is any point at infinity in $\mathbf{D}_{j}$ such that $\left|\operatorname{Re} \xi\left(c_{j}\right)\right|=\infty$. The error bounds (5.10) are valid at all points $z$ which can be joined to $c_{j}$ by a $\xi$-progressive path lying in $\mathbf{D}$. Finally, provided that the $c_{j}$ can be joined in pairs by paths of this kind, the fundamental connection formula between the $w_{j}(z)$ is expressed by (5.16) and (5.17).

Remark. It will be observed that an outstanding problem in the application of the connection formula (5.16) will be the determination of suitable $\xi$-progressive paths linking together the points at infinity $c_{j}$. This appears to be an unavoidable feature of this method, ${ }^{4}$ and its solution will generally necessitate a study of the actual conformal transformation between the $\xi$ and $z$-planes.

Usually it will not be difficult to demonstrate the existence of suitable $\xi$-progressive paths. These paths are not unique, however, and the most effective use of the connection formula clearly calls for the determination of those paths which minimize the total variation of the function $F$ along their length. Roughly speaking, this means that we need to keep the $\xi$-progressive paths as far away as possible from all the singularities of $f(z)$ and $h(z)$ and zeros of $f(z)$ (including $\left.z_{0}\right)$, subject to fulfillment of the monotonicity condition.

The construction of $\xi$-progressive paths in the neighborhood of infinity is discussed in section 7 below, and an example of the determination of $\xi$-progressive paths which minimize the variation of an analytic function along their length is given in [17], section 7.

\section{Airy's Equation}

The simplest example to which the theory of section 5 is applicable is provided by the differential equation

$$
d^{2} w / d z^{2}=z w
$$

In the notation of sections 4 and 5 , we take $f(z)=z$ and $h(z)=0$. Then $z_{0}=0$ and

$$
\xi(z)=\frac{2}{3} z^{3 / 2}, \quad g(z)=z^{-1 / 4}
$$

$\mathbf{D}$ comprises the whole $z$-plane, and the principal subdomains $\mathbf{D}_{j}$ are the sectors of angle $2 \pi / 3$ indicated in figure 3 .

We define $g_{1}(z)$ to be the principal branch of $z^{-1 / 4}$. Then from (5.08)

$$
g_{2}(z)=e^{\pi i / 6} z^{-1 / 4} \quad\left(z \in \mathbf{D}_{1} \cup \mathbf{D}_{2}\right), \quad g_{3}(z)=e^{-\pi i / 6} z^{-1 / 4} \quad\left(z \in \mathbf{D}_{1} \cup \mathbf{D}_{3}\right) .
$$




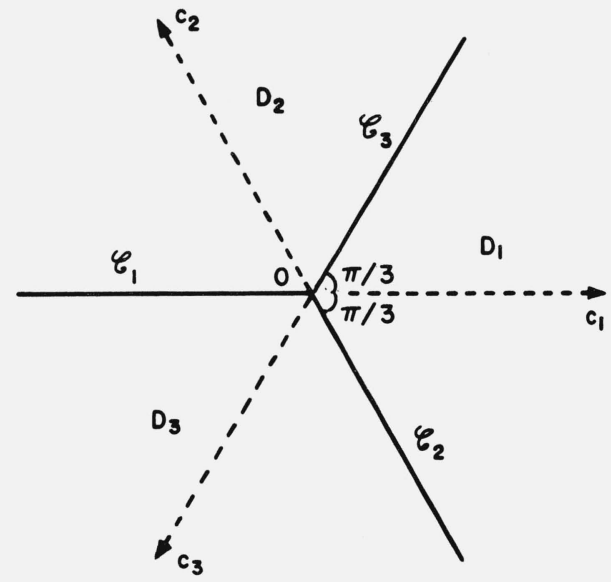

Figure 3. Principal subdomains for Airy's equation.

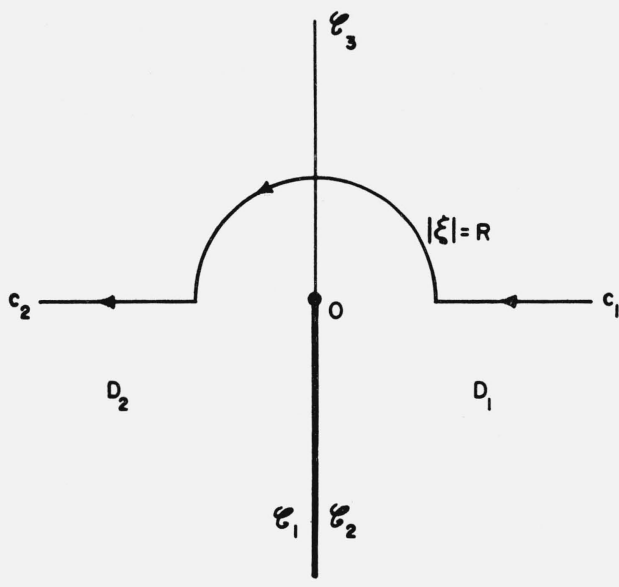

Figure 4. $\xi$-plane.

We take $c_{1}=+\infty, c_{2}=\infty e^{2 \pi i / 3}, c_{3}=\infty e^{-2 \pi i / 3}$. By considering the $\xi$-map we see that each region $\mathbf{H}\left(c_{j}\right)$ comprises the part of the $z$-plane remaining after the removal of the corresponding principal $\operatorname{curve}^{\mathscr{C}}{ }_{j}$. From (4.07) we have

$$
F(z)=\frac{5}{24 z^{3 / 2}}=\frac{5}{36 \xi}
$$

The $\xi$-map of a suitable $\xi$-progressive path linking $c_{1}$ with $c_{2}$ is shown in figure 4 . As the radius $R$ of the semicircular part tends to infinity, the variation of $F$ along the whole path vanishes. Therefore from (5.17) $\epsilon_{1}\left(c_{2}\right)=0$. Similarly $\epsilon_{2}\left(c_{3}\right)$ and $\epsilon_{3}\left(c_{1}\right)$ both vanish. Accordingly (5.16) reduces to

$$
w_{1}(z)+e^{-2 \pi i / 3} w_{2}(z)+e^{2 \pi i / 3} w_{3}(z)=0 .
$$

By comparing (5.09) with the asymptotic forms of the Airy functions [18], we identify

$$
w_{1}(z)=2 \pi^{1 / 2} \mathrm{Ai}(z), w_{2}(z)=2 \pi^{1 / 2} \mathrm{Ai}\left(z e^{-2 \pi i / 3}\right), \quad w_{3}(z)=2 \pi^{1 / 2} \mathrm{Ai}\left(z e^{2 \pi i / 3}\right) .
$$

Equation (6.05) is the well-known connection formula for these functions. It is interesting to observe that our theory yields it exactly.

The vanishing of the error terms in this example is essentially a consequence of there being no boundary of $\mathbf{D}$ intervening between $c_{j}$ and $c_{k}$ in the neighborhood of infinity. The path of figure 4 generally cannot be used to establish this in other cases. Using different paths, we formulate sufficient conditions for the vanishing of $\epsilon_{j}\left(c_{k}\right)$ in the next section.

\section{Sufficient Conditions for the Vanishing of the Error Terms}

We again use the notation of section 5 , and we suppose, without loss of generality, that the turning point $z_{0}$ is at the origin.

THEOREM 4. Let $f(z)$ and $h(z)$ be regular in the sector $\mathbf{S}: \gamma_{1}<\arg z<\gamma_{2}$ for all sufficiently large $|z|$, and

$$
f(z) \sim A z^{r}, \quad h(z)=O\left(z^{(r / 2)-1-\delta}\right), \text { as }|z| \rightarrow \infty,
$$

uniformly with respect to $\arg z$ in $\mathbf{S}$. Here $A$ is a real or complex non-zero constant, and $r, \delta$ real 
constants such that ${ }^{5} r>-2, \delta>0$. Also, within $\mathbf{S}$, let $\mathscr{L}_{1}, \mathscr{L}_{2}$ be outward-drawn rays from the origin which lie in adjacent principal subdomains $\mathbf{D}_{1}, \mathbf{D}_{2}$, respectively. Then provided that $\mathscr{L}_{1}, \mathscr{L}_{2}$ are not parallel to the asymptotes of the neighboring boundaries of $\mathbf{D}_{1}, \mathbf{D}_{2}$, they are strictly $\xi$-progressive paths in the neighborhood of infinity, and if $c_{1}, c_{2}$ denote the points at infinity on them, $\epsilon_{1}\left(c_{2}\right)=0$.

To prove this result, we first observe that

$$
g(z) \equiv\{f(z)\}^{-1 / 4} \sim A^{-1 / 4} z^{-r / 4}
$$

as $|z| \rightarrow \infty$ in S. Hence ([10], section 1.36)

$$
g^{\prime \prime}(z) \sim \frac{r}{4}\left(\frac{r}{4}+1\right) A^{-1 / 4} z^{-(r / 4)-2} \quad(r \neq 0)
$$

as $|z| \rightarrow \infty$ in any sector $\mathbf{S}^{\prime}: \gamma_{1}^{\prime}<\arg z<\gamma_{2}^{\prime}$ which is properly interior to $\mathbf{S}$. When $r=0$, the result corresponding to (7.03) is $g^{\prime \prime}(z)=o\left(z^{-2}\right)$. Therefore, whether or not $r$ is zero, we have

$$
g(z) g^{\prime \prime}(z)=O\left(z^{-s-1}\right)
$$

as $|z| \rightarrow \infty$ in $\mathbf{S}^{\prime}$, uniformly with respect to $\arg z$, where

$$
s=\frac{1}{2} r+1,
$$

and is positive, by hypothesis. From (4.07), (7.01), and (7.04) we derive

$$
F^{\prime}(z)=O\left(z^{-1-\delta_{1}}\right)
$$

where $\delta_{1}=\min (\delta, s)$. Hence the integral in (5.17) converges. The essential problem is to show how to construct a $\xi$-progressive path connecting $c_{1}$ with $c_{2}$ in the neighborhood of infinity.

From (5.01), we have

$$
\xi=\frac{A^{1 / 2} z^{s}}{s}+o\left(z^{s}\right) \quad(z \in \mathbf{S})
$$

Therefore the principal curves approach infinity in directions parallel to the rays

$$
\arg z=\left\{\left(n+\frac{1}{2}\right) \pi-\frac{1}{2} \alpha\right\} / s,
$$

where $\alpha=\arg A$, and $n=0, \pm 1, \pm 2, \ldots$ We call these the principal directions. Consider the behavior of $\operatorname{Re} \xi$ along the ray with parametric equation $z=\tau e^{i \gamma}, \gamma$ being real and fixed, and $0<\tau<\infty$. We have

$$
d \xi / d \tau=\{f(z)\}^{1 / 2}(d z / d \tau)=A^{1 / 2} \tau^{r / 2} e^{i s \gamma}\{1+o(1)\}
$$

as $\tau \rightarrow \infty$. Hence

$$
d(\operatorname{Re} \xi) / d \tau=|A|^{1 / 2} \tau^{r / 2}\left\{\cos \left(s \gamma+\frac{1}{2} \alpha\right)+o(1)\right\} .
$$

The constant $\cos \left(s \gamma+\frac{1}{2} \alpha\right)$ vanishes only when $\gamma$ has one of the values given by the right of (7.07). Therefore any outward ray in $\mathbf{S}$ not in a principal direction is a strictly $\xi$-progressive path for all sufficiently large $|z|$. In particular this includes $\mathscr{L}_{1}$ and $\mathscr{L}_{2}$.

\footnotetext{
5 Thus the differential equation (4.01) has an irregular singularity at infinity.
} 


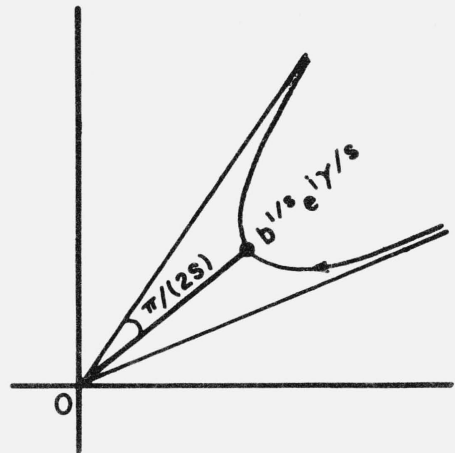

Figure 5. z-plane.

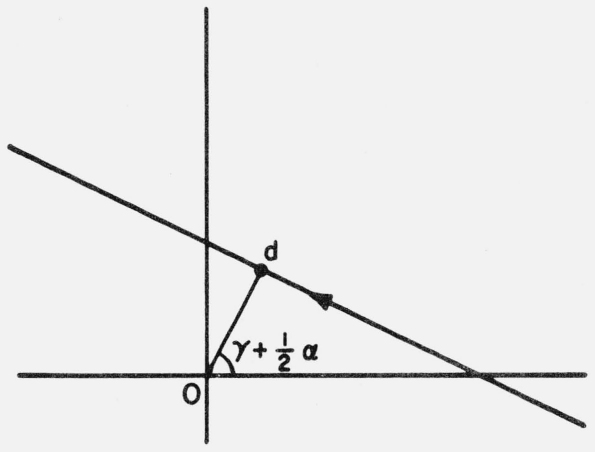

FIGURE 6. Approximate path in $\xi$-plane: $|d|=|A|^{1 / 2} b / s$.

Next, consider the path with parametric equation

$$
z=\left\{(b+i \tau) e^{i \gamma}\right\}^{1 / s_{i}} \quad(-\infty<\tau<\infty)
$$

where $\gamma$ and $b$ are real constants, $b$ being large and positive.

As indicated in figure 5 this path begins at infinity, sweeps back to its nearest approach to the origin at $z=b^{1 / s} e^{i \gamma / s}$ and then returns to infinity, the angle between its asymptotes being $\pi / s$. When $b$ is large, we have from (7.06)

$$
\xi \sim s^{-1}|A|^{1 / 2}(b+i \tau) e^{i\{\gamma+(\alpha / 2)\}},
$$

showing that the $\xi$-map of (7.10) is approximately the straight line indicated in figure 6 . Corresponding to (7.09), we find that

$$
\frac{d}{d \tau}(\operatorname{Re} \xi)=-\frac{|A|^{1 / 2}}{s}\left\{\sin \left(\gamma+\frac{1}{2} \alpha\right)+o(1)\right\},
$$

as $b \rightarrow \infty$, uniformly with respect to $\tau$. Therefore the curve (7.10) is a strictly $\xi$-progressive path for all sufficiently large $b$, except when its asymptotes are in principal directions.

Now consider $c_{1}$ and $c_{2}$. By taking $\gamma_{1}^{\prime}$ and $\gamma_{2}^{\prime}$ sufficiently close to $\gamma_{1}$ and $\gamma_{2}$, respectively, we can ensure that $\mathbf{S}^{\prime}$ contains $\mathscr{L}_{1}$ and $\mathscr{L}_{2}$. From (7.06) and the hypotheses on $\mathscr{L}_{1}$ and $\mathscr{L}_{2}$, it follows that

$$
\left|\arg c_{2}-\arg c_{1}\right|<2 \pi / s \text {. }
$$

Figure 7 indicates how $c_{1}$ and $c_{2}$ can be connected by a strictly $\xi$-progressive path in $\mathbf{S}^{\prime}$, consisting of $\mathscr{L}_{1}, \mathscr{L}_{2}$ and either one or two arcs of the type (7.10), depending whether or not $\left|\arg c_{2}-\arg c_{1}\right|$ $<\pi / s$. The corresponding path in the $\xi$-plane is approximately that indicated in figure 8 . On letting the $b$-parameters of the arcs tend to infinity, we see immediately from (7.05) that the variations of $F$ along $\mathscr{L}_{1}$ and $\mathscr{L}_{2}$ tend to zero. For the contribution to the variation from the arcs (7.10), we observe that the corresponding values of $\int\left|z^{-1-\delta_{1}} d z\right|$ are bounded by

$$
\frac{1}{s} \int_{-\infty}^{\infty} \frac{d \tau}{|b+i \tau|^{1+\left(\delta_{1} / s\right)}}
$$

and accordingly vanish as $b \rightarrow \infty$. The proof of Theorem 4 is now complete.

An alternative set of sufficient conditions for the vanishing of the error terms is provided by the next theorem, which can be proved in a similar way. In this result $m, A$ denote real or complex nonzero constants, and $\delta$ is a positive constant. 


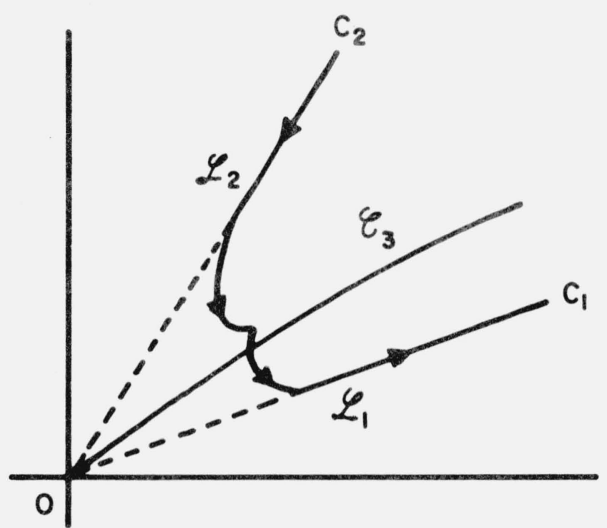

FiguRE 7. $\xi$-progressive path in z-plane.

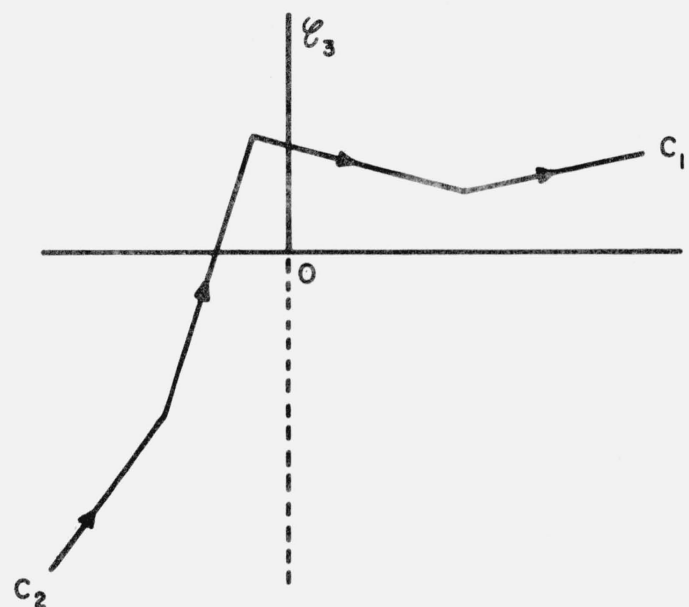

FiguRE 8. Approximate path in $\xi$-plane.

THEOREM 5. Let $f(z)$ and $h(z)$ be regular in the strip $\mathbf{T}: \gamma_{1}<\operatorname{Im}(m z)<\gamma_{2}$ for all sufficiently large positive values of $\operatorname{Re}(m z)$, and

$$
f(z) \sim A e^{m z}, \quad h(z)=O\left(e^{m z(1-\delta) / 2}\right), \text { as } \operatorname{Re}(m z) \rightarrow+\infty,
$$

uniformly with respect to $\operatorname{Im}(m z)$ in $\mathbf{T}$. Also, within $\mathbf{T}$, let $\mathscr{L}_{1}, \mathscr{L}_{2}$ be straight lines which are parallel to the boundaries of $\mathbf{T}$, and lie in adjacent principal subdomains $\mathbf{D}_{1}, \mathbf{D}_{2}$, respectively. Then provided that $\mathscr{L}_{1}, \mathscr{L}_{2}$ do not coincide with the asymptotes of the boundaries of $\mathbf{D}_{1}, \mathbf{D}_{2}$, they are strictly $\xi$-progressive paths in the neighborhood of infinity, and if $c_{1}, c_{2}$ are the points at infinity on them, $\epsilon_{1}\left(c_{2}\right)=0$.

\section{The Gans-Jeffreys Connection Formulas (i)}

The first of these two formulas may be regarded as a special case of the fundamental connection formula of section 5. We again consider the differential equation (4.01) in which $f(z)$ and $h(z)$ are regular in an unbounded, simply connected domain $\mathbf{D}$ and $f(z)$ has just one zero in $\mathbf{D}$, a simple zero at $z=0$. This time we suppose that $\mathbf{D}$ includes the whole of the real axis, and that $f(z)$ and $h(z)$ are real when $z$ is real. Without loss of generality, we take $f(z) / z$ to be positive on the real axis.

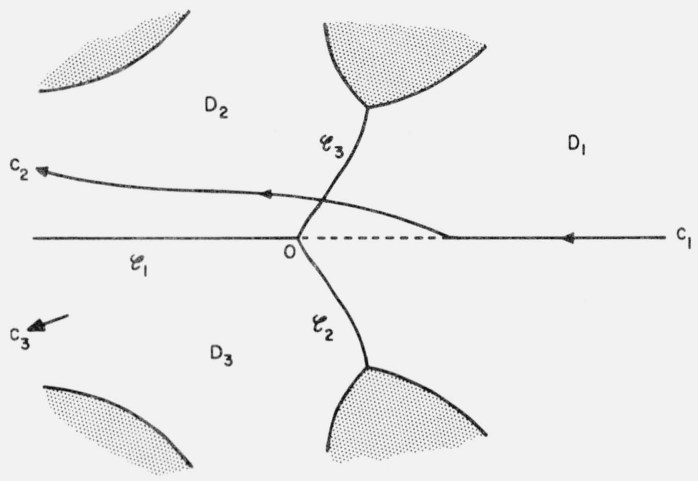

Figure 9. Typical principal subdomains $\mathbf{D}_{j}$ for the Gans-Jeffreys formulas. 
One of the three principal curves, say $\mathscr{C}_{1}$, which emerges from the origin is the negative real axis. The other two emerge at directions $\pm \pi / 3$ and are symmetric with respect to the real axis. As in section 5 we denote by $\mathbf{D}_{1}, \mathbf{D}_{2}, \mathbf{D}_{3}$ the three subdomains bounded by the principal curves which have the origin on their boundary.

We take $c_{1}$ to be the point at infinity on the positive real axis, and $c_{2}, c_{3}$ to be points at infinity on conjugate $\xi$-progressive curves $\mathscr{L}_{2}, \mathscr{L}_{3}$. These curves must lie within $\mathbf{D}_{2}, \mathbf{D}_{3}$, respectively, and be such that no boundary of $\mathbf{D}$ intervenes between them in the neighborhood of infinity. We assume that $|\operatorname{Re} \xi(z)| \rightarrow \infty$ when $z \rightarrow c_{j}(j=1,2,3)$ (the "dominancy condition") and that the variation of the function $F$, defined by (4.07), converges when $z \rightarrow c_{j}$. The other assumptions we need are

(i) $\xi$-progressive paths can be found in $\mathbf{D}$ which link $c_{2}$ or $c_{3}$ with any point of the real axis, other than the origin, and which do not pass through the origin.

(ii) $\epsilon_{2}\left(c_{3}\right), \epsilon_{2}(-\infty)$ and $\epsilon_{3}(-\infty)$ all vanish.

In applications, Condition (i) can be tested by considering the $\xi$-map. Sufficient sets of conditions for (ii) are to be found in section 7 .

The connection formula (5.16) for the solutions of (4.01) takes the form

$$
w_{1}(z)=e^{\pi i / 3}\left\{1+\epsilon_{3}\left(c_{1}\right)\right\} w_{2}(z)+e^{-\pi i / 3}\left\{1+\epsilon_{2}\left(c_{1}\right)\right\} w_{3}(z) .
$$

To fix the solutions completely, we define $g_{1}(z)$ to be the branch of $\{f(z)\}^{-1 / 4}$ which takes its principal value on the positive real axis (compare (5.08)). The branches of the $w_{j}(z)$ are then interpreted in real form as follows.

When $z$ is positive, we have immediately from (5.09) and (5.10)

$$
w_{1}(z)=f^{-1 / 4} \exp \left(-\int_{0}^{z} f^{1 / 2} d z\right)\left\{1+\epsilon_{1}(z)\right\} \quad(z>0),
$$

where

$$
\left|\epsilon_{1}(z)\right| \leqslant \exp \left\{\%_{z, x}(F)\right\}-1
$$

the variation being taken along the real axis.

When $z$ is negative, we find that

$$
\xi_{2}(z)=i \int_{z}^{0}|f|^{1 / 2} d z, \quad g_{2}(z)=e^{-\pi i / 12}|f|^{-1 / 4} .
$$

Substitution of (5.09) in (8.01) accordingly yields

$$
w_{1}(z)=\left\{1+\epsilon_{3}\left(c_{1}\right)\right\} e^{\pi i / 4}|f|^{-1 / 4} \exp \left(-i \int_{z}^{0}|f|^{1 / 2} d z\right)\left\{1+\epsilon_{2}(z)\right\}+\text { conjugate. }
$$

Let

$$
1+\epsilon_{2}(z)=\{1+\mu(z)\} e^{-i \eta(z)}, \quad 1+\epsilon_{3}(z)=\{1+\mu(z)\} e^{i \eta(z)},
$$

where $\mu(z)$ and $\eta(z)$ are real and $\mu(z) \geqslant-1$. Then by elementary considerations

$$
|\mu(z)| \leqslant\left|\epsilon_{2}(z)\right|, \quad|\eta(z)| \leqslant \sin ^{-1}\left|\epsilon_{2}(z)\right| \leqslant \pi\left|\epsilon_{2}(z)\right| / 2,
$$

provided that $\left|\epsilon_{2}(z)\right| \leqslant 1$. Substitution in (8.05) yields

$$
w_{1}(z)=2\left\{1+\mu\left(c_{1}\right)\right\}\{1+\mu(z)\}|f|^{-1 / 4} \sin \left\{\int_{z}^{0}|f|^{1 / 2} d z+\frac{1}{4} \pi+\eta(z)-\eta\left(c_{1}\right)\right\} \quad(z<0) .
$$


From (8.07), (5.17) and (5.10), we deduce that

$$
\left|\mu\left(c_{1}\right)\right|,\left|2 \eta\left(c_{1}\right) / \pi\right| \leqslant \frac{1}{2}\left[\exp \left\{\psi_{c_{1}, c_{2}}(F)\right\}-1\right],
$$

and

$$
|\mu(z)|,|2 \eta(z) / \pi| \leqslant \exp \left\{\mathscr{\gamma}_{z, c_{2}}(F)\right\}-1,
$$

the variations in both cases being evaluated along $\xi$-progressive curves.

The aggregate of (8.02), (8.03), (8.08), (8.09), and (8.10), may be regarded as the full form of the first Gans-Jeffreys connection formula [5], [6], [9]. It expresses the subdominant exponential solution on one side of the turning point in terms of the oscillatory solutions on the other side. Boundary conditions satisfied by $w_{1}(z)$ are clearly

$$
\epsilon_{1}(z)=o(1) \quad(z \rightarrow+\infty) ; \quad \mu(z), \eta(z)=o(1) \quad(z \rightarrow-\infty) .
$$

We note that the bounds for the error terms $\mu\left(c_{1}\right), \eta\left(c_{1}\right)$ depend solely on the variation of $F$ along the $\xi$-progressive path linking $c_{1}$ and $c_{2}$, and can be minimized by proper choice of path.

The second connection formula concerns the dominant exponential solution. Unlike the subdominant solution, this is not unique. Consider first $w_{2}(z)$. For negative $z$, we have from (8.04) and (5.09)

$$
w_{2}(z)=e^{-\pi i / 12}|f|^{-1 / 4} \exp \left(-i \int_{z}^{0}|f|^{1 / 2} d z\right)\left\{1+\epsilon_{2}(z)\right\} \quad(z<0)
$$

On the other side of the turning point

$$
w_{2}(z)=e^{\pi i / 6} f^{-1 / 4} \exp \left(\int_{0}^{z} f^{1 / 2} d z\right)\left\{1+\epsilon_{2}(z)\right\} \quad(z>0) .
$$

Instead of $w_{2}(z)$, however, we prefer the real solution $w_{4}(z)=\operatorname{Re}\left\{e^{-\pi i / 6} w_{2}(z)\right\}$. Then with the aid of (8.06), we derive

$$
w_{4}(z)=\left[1+\operatorname{Re}\left\{\epsilon_{2}(z)\right\}\right] f^{-1 / 4} \exp \left(\int_{0}^{z} f^{1 / 2} d z\right) \quad(z>0),
$$

and

$$
w_{4}(z)=\{1+\mu(z)\}|f|^{-1 / 4} \cos \left\{\int_{z}^{0}|f|^{1 / 2} d z+\frac{1}{4} \pi+\eta(z)\right\} \quad(z<0)
$$

where $\left|\epsilon_{2}(z)\right|,|\mu(z)|$ and $|2 \eta(z) / \pi|$ are all bounded by the right of (8.10). These relations constitute the second Gans-Jeffreys formula. -We note that $w_{4}(z)$ satisfies the boundary conditions

$$
\epsilon_{2}(z)=\epsilon_{2}\left(c_{1}\right)+o(1) \quad(z \rightarrow+\infty) ; \quad \mu(z), \eta(z)=o(1) \quad(z \rightarrow-\infty) ;
$$

where $\left|\epsilon_{2}\left(c_{1}\right)\right|$ is bounded by the right of (8.09).

\section{The Gans-Jeffreys Connection Formulas (ii)}

In this section we attack the problem of section 8 with real-variable theory. Our hypotheses are

(i) The function $f(z) / z$ is positive and twice-continuously differentiable throughout the interval $-\infty<z<\infty$, and $h(z)$ is a continuous real function in the same interval. 
(ii) As $z \rightarrow \pm \infty,\left|\int_{0}^{z} f^{1 / 2} d z\right|$ is unbounded and the variation of the function $F$ (defined by (4.07)) converges.

In these circumstances it is shown in [15], sections 4,5 that (4.01) has a solution ${ }^{6}$

$$
w_{1}(z)=(\zeta / f)^{1 / 4}\left\{\operatorname{Ai}(\zeta)+\epsilon_{1}(z)\right\}
$$

where

$$
\begin{gathered}
\zeta \equiv \zeta(z)= \pm\left|\frac{3}{2} \int_{0}^{z}\{f(t)\}^{1 / 2} d t\right|^{2 / 3} \\
\left|\epsilon_{1}(z)\right| \leqslant \lambda_{1}^{-1} E^{-1}(\zeta) M(\zeta)\left[\exp \left\{\lambda_{1} \mathscr{V}_{z, \infty}(H)\right\}-1\right] \quad(-\infty<z<\infty),
\end{gathered}
$$

and

$$
H(z)=\frac{5}{24|\zeta|^{3 / 2}} \pm \int^{z}\left\{\frac{1}{|f|^{1 / 4}} \frac{d^{2}}{d z^{2}}\left(\frac{1}{|f|^{1 / 4}}\right)-\frac{h}{|f|^{1 / 2}}\right\} d z
$$

The upper or lower signs are taken in (9.02) and (9.04) according as $z$ is positive or negative, and the integration limits in (9.04) are determined by the condition $H(0)=0$. The auxiliary functions $E(\zeta)$, $M(\zeta)$ and the constant $\lambda_{1}=1.430 \ldots$ are defined in [15], section 2.

On substituting for $\mathrm{Ai}(\zeta)$ by means of its appropriate asymptotic forms, we obtain

$$
w_{1}(z)=\frac{1}{2} \pi^{-1 / 2} f^{-1 / 4}\{1+o(1)\} \exp \left(-\int_{0}^{z} f^{1 / 2} d z\right) \quad(z \rightarrow+\infty),
$$

and

$$
w_{1}(z)=\pi^{-1 / 2}|f|^{-1 / 4}\left[\sin \left(\int_{z}^{0}|f|^{1 / 2} d z+\frac{1}{4} \pi\right)+\rho(z)+o(1)\right] \quad(z \rightarrow-\infty),
$$

where

$$
\rho(z)=\pi^{1 / 2}|\zeta|^{1 / 4} \epsilon_{1}(z)
$$

and is bounded.

In order to throw (9.06) fully into "modulus-phase" form, we appeal to Theorem 4 of [1]. This shows that solutions of (4.01) exist of the form

$$
|f|^{-1 / 4}\{1+o(1)\} \exp \left\{ \pm i \int_{z}^{0}|f|^{1 / 2} d z\right\} \text { as } z \rightarrow-\infty \text {. }
$$

Therefore we can express

$$
w_{1}(z)=\pi^{-1 / 2}|f|^{-1 / 4}\left\{1+\nu_{1}+o(1)\right\} \sin \left\{\int_{z}^{0}|f|^{1 / 2} d z+\frac{1}{4} \pi+\nu_{2}+o(1)\right\} \quad(z \rightarrow-\infty),
$$

where $\nu_{1}, \nu_{2}$ are real constants to be determined. Comparison of (9.06) with (9.09) shows that

$$
\rho(z)=\left(1+\nu_{1}\right) \sin \left\{\theta(z)+\nu_{2}\right\}-\sin \theta(z)+o(1),
$$

${ }^{6}$ Not to be confused with the $w_{1}(z)$ of section 8 . 
where

$$
\theta(z) \equiv \int_{z}^{0}|f|^{1 / 2} d z+\frac{1}{4} \pi
$$

Hence $\rho(z)$ has the form

$$
\rho(z)=\rho_{L} \sin \left\{\theta(z)+\nu_{3}\right\}+o(1),
$$

where $\rho_{L}$ and $\nu_{3}$ are real constants $\left(\rho_{L} \geqslant 0\right)$ related to $\nu_{1}$ and $\nu_{2}$. Clearly

$$
\rho_{L}=\varlimsup_{z \rightarrow-\infty}|\rho(z)|
$$

Accordingly, from (9.03), (9.07) and the fact that $|\zeta|^{1 / 4} M(\zeta) \rightarrow \pi^{-1 / 2}$ as $\zeta \rightarrow-\infty$, we have

$$
\rho_{L} \leqslant \lambda_{1}^{-1}\left[\exp \left\{\lambda_{1} \mathscr{V}_{-x, x}(H)\right\}-1\right]
$$

In (9.10) and (9.12) let $z \rightarrow-\infty$ through a sequence of values for which $\theta(z)$ is an integral multiple of $\pi$ (compare hypothesis (ii) above). This gives

$$
\left(1+\nu_{1}\right) \sin \nu_{2}=\rho_{L} \sin \nu_{3}
$$

Alternatively, by using a sequence of odd integral multiples of $\frac{1}{2} \pi$ we derive

$$
\left(1+\nu_{1}\right) \cos \nu_{2}-1=\rho_{L} \cos \nu_{3}
$$

Combination of (9.15) and (9.16) yields

$$
\left(1+\nu_{1}\right) e^{i \nu_{2}}=1+\rho_{L} e^{i \nu_{3}}
$$

Therefore

$$
\left|\nu_{1}\right| \leqslant \rho_{L}, \quad\left|\nu_{2}\right| \leqslant \sin ^{-1} \rho_{L} \leqslant \pi \rho_{L} / 2
$$

provided that $\rho_{L} \leqslant 1$ (compare (8.07)).

The first Gans-Jeffreys formula is represented by the aggregate of (9.05), (9.09), (9.14), and (9.18).

A second solution of (4.01) is [15]

$$
w_{2}(z)=(\zeta / f)^{1 / 4}\left\{\operatorname{Bi}(\zeta)+\epsilon_{2}(z)\right\}
$$

where

$$
\left|\epsilon_{2}(z)\right| \leqslant\left(\lambda_{2} / \lambda_{1}\right) E(\zeta) M(\zeta)\left[\exp \left\{\lambda_{1} \mathscr{V}_{-\infty, z}(H)\right\}-1\right]
$$

and $\lambda_{2}=1.315 \ldots$ Substitution of the asymptotic forms for $\operatorname{Bi}(\zeta)$ yields

$$
w_{2}(z)=\pi^{-1 / 2} f^{-1 / 4}\{1+\sigma(z)+o(1)\} \exp \left(\frac{2}{3} \zeta^{3 / 2}\right) \quad(z \rightarrow+\infty)
$$


and

$$
w_{2}(z)=\pi^{-1 / 2}|f|^{-1 / 4}\left\{\cos \left(\frac{2}{3}|\zeta|^{3 / 2}+\frac{1}{4} \pi\right)+o(1)\right\} \quad(z \rightarrow-\infty)
$$

where

$$
\sigma(z)=\pi^{1 / 2} \zeta^{1 / 4} \exp \left(-\frac{2}{3} \zeta^{3 / 2}\right) \epsilon_{2}(z)
$$

An obvious extension of Theorem 2 of the present paper shows that $\sigma(z)$ tends to a finite limit, $\sigma(\infty)$, as $z \rightarrow+\infty$. Accordingly

$$
w_{2}(z)=\pi^{-1 / 2} f^{-1 / 4}\{1+\sigma(\infty)+o(1)\} \exp \left(\int_{0}^{z} f^{1 / 2} d z\right) \quad(z \rightarrow+\infty) .
$$

From (9.20) and the asymptotic form of $M(\zeta)$, we obtain

$$
|\sigma(\infty)| \leqslant \frac{1}{2} \sqrt{5} \lambda_{2} \lambda_{1}^{-1}\left[\exp \left\{\lambda_{1} \mathscr{V}_{-\infty, \infty}(H)\right\}-1\right]
$$

And with the aid of analysis similar to that used for throwing (9.06) into the form (9.09), we readily show that

$$
w_{2}(z)=\pi^{-1 / 2}|f|^{-1 / 4}\{1+o(1)\} \cos \left\{\int_{z}^{0}|f|^{1 / 2} d z+\frac{1}{4} \pi+o(1)\right\} \quad(z \rightarrow-\infty) .
$$

Relations (9.24), (9.25), and (9.26) consititute the second Gans-Jeffreys connection formula.

Sharper bounds. Somewhat improved bounds for the error terms of this section can be achieved by application of Theorem 3 of [15], , and, in the case of the Bi-type solution, of an inequality of the same character as (3.03) above. The results are expressed by

$$
\rho_{L} \leqslant \exp \left\{\mathscr{V}_{-\infty, 0}(H)+\lambda_{4} \mathscr{V}_{0, \infty}(H)\right\}-1
$$

and

$$
|\sigma(\infty)| \leqslant \exp \left\{\frac{2}{\sqrt{3}} \mathscr{V}_{-x, 0}(H)+\lambda_{5} \mathscr{V}_{0, x}(H)\right\}-1-\left(\frac{2}{\sqrt{3}}-\frac{1}{2}\right) \mathscr{V}_{-\infty, 0}(H)
$$

where $\lambda_{4}=0.521 \ldots$ and $\lambda_{5}=0.520 \ldots$

\section{Summary}

The primary purpose of this paper has been to investigate approximate solutions of the differential equations (1.01) and, more generally, (4.01) in a complex domain containing no singularities of the functions $f(z)$ and $h(z)$, and a simple zero of $f(z)$. Some preliminary results concerning the Liouville-Green approximation are given in sections 2 through 4 . The connection formula for the three fundamental approximations of this kind is then established in section 5 , complete with strict error bounds, by considering limiting behavior at the point at infinity. An illustrative application to Airy's differential equation is made in section 6 , and some general circumstances in which the error terms vanish are investigated in section 7.

An important special case concerns real variables: the problem here is to connect the exponential-type solutions on one side of the turning point with the oscillatory solutions on the other. 
This is solved in section 8 by use of the general formula of section 5 . An alternative solution is constructed in section 9 solely from real-variable theory, by re-expansion of known uniform Airyfunction approximations. Naturally, different hypotheses on $f(z)$ and $h(z)$ are made in the two methods, but the principal difference lies in the forms of the error bounds. In the first method the bounds depend on the variation of a certain function along an infinite path of specified type in the complex plane. In the second method the bounds depend on the variation of a more complicated function along the real axis.

The error bounds given in this paper have computational applications. As shown in the paper which follows [19], they may also be used to provide theoretical insight into the accuracy of the connection formulas in various circumstances, including cases when the differential equation contains a large parameter. Hitherto, in certain applications it has often been difficult to assess the validity of assumptions which have been made.

\section{Addendum}

The problems studied in the present paper and its sequel [19] have also been considered in a recent monograph by Fröman and Fröman [20]. These authors have the same principal objective, that is, the determination of strict error bounds for the coefficients in the connection formulas. The method they use resembles the first method of the present paper in that it is a rigorous formulation of the procedure of Zwaan. The difference in the approaches is as follows.

We constructed (in sec. 5) the connection formula for the three fundamental Liouville-Green solutions associated with a simple turning point of the differential equation (4.01) by examining the asymptotic behavior of these solutions in the neighborhood of the point at infinity. Fröman and Fröman, following Kemble [3], express the general solution of (4.01) in the form

$$
w(z)=a_{1}(z) f^{-1 / 4} \exp \left(\int f^{1 / 2} d z\right)+a_{2}(z) f^{-1 / 4} \exp \left(-\int f^{1 / 2} d z\right),
$$

and derive a pair of first-order simultaneous differential equations for the functions $a_{1}(z)$ and $a_{2}(z)$. These equations are then used to determine the changes in these functions as $z$ passes from one principal subdomain to another. Error bounds for the changes are expressed in terms of contour integrals which are equivalent to the variations of our function $F(z)$.

Generally the path for the contour integrals consists of the real $z$-axis with indentations by. passing the turning points. Fröman and Fröman show that the appropriate form of monotonicity condition is fulfilled when the indentations are semicircles of sufficiently small radius ([20], p. 39). Owing to the singularities of $F(z)$ at the turning points however, the error bounds tend to infinity as the indentations shrink to zero. In consequence Fröman and Fröman assume that the indentations can actually be taken sufficiently large to yield small error bounds, without violating the monotonicity condition. In the present paper we allow greater flexibility in the choice of paths, with a view to achieving minimum bounds in applications.

Other comparisons with the first method of the present paper are as follows:

(i) There is greater emphasis in [20] on the case in which the coefficients $f(z)$ and $h(z)$ in the differential equation (4.01) are real on the real axis.

(ii) Approximations and expansions are frequently used in [20] to simplify the error bounds. There is greater emphasis on strict bounds in the present paper and [19].

(iii) In the special limiting case in which $z$ is at infinity the error bounds can be sharpened by a factor of 2 (see Theorem 2 of sec. 3 above). This refinement is freely used in the present paper and [19], but is not in evidence in [20].

The author acknowledges helpful criticisms of the draft of this paper by Drs. J. Heading, L. Maximon, and F. Stenger.

The work described above has been supported by the U.S. Army Research Office, Durham, North Carolina (Project No. 4238-M). 


\section{References}

[1] Olver, F. W. J., Error bounds for the Liouville-Green (or WKB) approximation, Proc. Camb. Phil. Soc. 57, 790-810 (1961).

[2] Zwaan, A., Intensitäten im Ca-Funkenspektrum, Arch. Néerlandaises des Sciences Exactes, Ser. 3A 12, 1-76 (1929).

[3] Kemble, E. C., A contribution to the theory of the BWK method, Phys. Rev. 48, 549-561 (1935).

[4] Furry, W. H., Two notes on phase-integral methods, Phys. Rev. 71, 360-371 (1947).

[5] Gans, R., Fortpflanzung des Lichts durch ein inhomogenes Medium, Ann. Phys. Lpz. 47, $709-736$ (1915).

[6] Jeffreys, H., On certain approximate solutions of linear differential equations of the second order, Proc. Lond. Math. Soc. (2) 23, 428-436 (1924-5).

[7] Kramers, H. A., Wellenmechanik und halbzahlige Quantisierung, Z. Phys. 39, 828-840 (1926).

[8] Langer, R. E., On the connection formulas and the solutions of the wave equation, Phys. Rev. 51, 669-676 (1937).

[9] Jeffreys, H., On approximate solutions of linear differential equations, Proc. Camb. Phil. Soc. 49, 601-611 (1953).

[10] Jeffreys, H., Asymptotic approximations (Oxford University Press, 1962).

[11] Morse, P. M. and Feshbach, H., Methods of theoretical physics, Pt 2 (McGraw-Hill Book Co., Inc., New York, N.Y. 1953).

[12] Budden, K. G., Radio waves in the ionosphere. (Cambridge University Press, 1961).

[13] Heading, J., An introduction to phase-integral methods, (John Wiley \& Sons, Inc., New York, N.Y., 1962).

[14] Langer, R. E., The asymptotic solutions of ordinary linear differential equations of the second order, with special reference to the Strokes phenomenon, Bull. Amer. Math. Soc. 40, 545-582 (1934).

[15] Olver, F. W. J., Error bounds for first approximations in turning-point problems, J. Soc. Indust. Appl. Math. 11, 748772 (1963).

[16] Olver, F. W. J., Uniform asymptotic expansions of solutions of linear second-order differential equations for large values of a parameter, Phil. Trans. Roy. Soc. London, Ser. A 250, 479-517 (1958).

[17] Olver, F. W. J., On the asymptotic solutions of second-order differential equations having an irregular singularity of rank one, with an application to Whittaker functions, J. Soc. Indust. Appl. Math. Ser. B 2, 225-243 (1965).

[18] Miller, J. C. P., The Airy Integral, Brit. Ass. Math. Tab. Part-Vol. B. (Cambridge University Press, 1946).

[19] Olver, F. W. J., Error analysis of phase-integral methods. II. Application to wave-penetration problems, J. Res. NBS 69B, No. 4, 291-300 (1965).

[20] Fröman, N. and Fröman, P. O., JWKB approximation-Contributions to the theory (North-Holland Publishing Co., Amsterdam, 1965).

(Paper 69B4-157) 